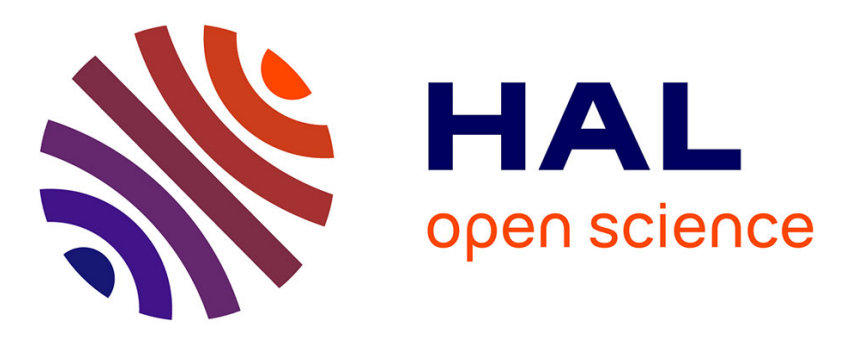

\title{
Influence of an amorphous surface layer on the mechanical properties of metallic nanoparticles under compression
}

Alexandra Goryaeva, Claudio Fusco, Matthieu Bugnet, Jonathan Amodeo

\section{To cite this version:}

Alexandra Goryaeva, Claudio Fusco, Matthieu Bugnet, Jonathan Amodeo. Influence of an amorphous surface layer on the mechanical properties of metallic nanoparticles under compression. Physical Review Materials, 2019, 3 (3), 10.1103/PhysRevMaterials.3.033606 . hal-02078122

\section{HAL Id: hal-02078122 \\ https://hal.science/hal-02078122}

Submitted on 25 Mar 2019

HAL is a multi-disciplinary open access archive for the deposit and dissemination of scientific research documents, whether they are published or not. The documents may come from teaching and research institutions in France or abroad, or from public or private research centers.
L'archive ouverte pluridisciplinaire HAL, est destinée au dépôt et à la diffusion de documents scientifiques de niveau recherche, publiés ou non, émanant des établissements d'enseignement et de recherche français ou étrangers, des laboratoires publics ou privés. 


\title{
Influence of an amorphous surface layer on the mechanical properties of metallic nanoparticles under compression
}

\author{
Alexandra M. Goryaeva, ${ }^{*}$ Claudio Fusco, Matthieu Bugnet, and Jonathan Amodeo ${ }^{\dagger}$ \\ Université de Lyon, INSA-Lyon, MATEIS, UMR 5510 CNRS, 69621 Villeurbanne, France
}

(Received 21 December 2018; published 20 March 2019)

\begin{abstract}
This study aims to investigate the role of amorphous surface layers on the mechanical response of metallic nanoparticles under compression using molecular dynamics simulations. For this purpose, the transferability of three embedded-atom-method (EAM) potentials to model monoatomic Ni glass and amorphous-crystalline structures is examined. Particular attention is paid to the crystallisation rate of the amorphous shell surrounding the crystalline inner structure. Relying on the most appropriate model, the influence of the amorphous layer on the mechanical response of crystalline-amorphous Ni nanoparticles is further investigated. Regardless of its thickness, the amorphous surface layer significantly changes both the effective elastic modulus of the nanoparticle and the flow stress. Besides stress, dislocation nucleation processes as well as the final shape of the compressed particles are influenced by the presence of the amorphous shell. These results bring new insights on the influence of surface state on the mechanics of metallic nano-objects.
\end{abstract}

DOI: 10.1103/PhysRevMaterials.3.033606

\section{INTRODUCTION}

Nano-objects, such as nanoparticles (NPs), nanowires (NWs), and nanopillars are characterized by a large surface to volume ratio that promotes their exceptional chemical, physical and mechanical properties. Among the various nanoobjects, NPs are used in a wide range of functional applications, such as nanoelectromechanical systems (NEMS) [1,2], drug delivery systems and catalysts $[3,4]$, biological detectors and sensors $[5,6]$. They also make strong friction-reducer agents in lubricants [7,8]. For all these applications, the targeted functional property depends strongly on the shape, the thermomechanical history, and the surface state of the NP. Besides, nanocrystals exhibit particularly high elastic stretchability, increased yield strength (within the gigapascal range) and extended ductility, when compared to their bulk counterpart. These unique mechanical properties drive up NPs as particularly interesting building blocks for architectured material applications as well as for more fundamental studies.

The mechanical properties of metallic NPs are commonly investigated using transmission electron microscopy (TEM) or scanning electron microscopy (SEM) compression tests and computed using atomistic simulations [9-13]. It is worth noticing that most molecular dynamics (MD) studies consider perfectly shaped crystalline NPs, distinct from each other by size and facets geometry. Generally, the yield of metallic NPs is attributed to the nucleation of partial dislocations (and/or nanotwins) from the surfaces, edges, and corners. Recently, Amodeo and Lizoul [14] have shown the influence of smoothing the edges and corners of $\mathrm{Ni}_{3} \mathrm{Al}$ nanocubes on

\footnotetext{
* Present address: DEN-Service de Recherches de Métallurgie Physique, CEA Saclay, 91191 Gif-sur-Yvette, France;

alexandra.goryaeva@cea.fr

†jonathan.amodeo@insa-lyon.fr
}

the mechanical response using MD. This study emphasizes a particular increase of the yield strength for rounded systems due to a decrease of the contact surface area, and a transition towards a homogeneous dislocation nucleation process for a given range of sample sizes and roundness. Both results were confirmed by Sharma and collaborators [15], in the case of faceted Ni NPs using SEM compression tests and MD. In the latter case, the authors also underline the crucial role of the thin surface oxide layer (observed in the experiments) on the elevated strengths of the NPs. The question of the influence of the surface state (e.g., oxidation, amorphization) on the mechanical properties and dislocation nucleation processes at small scales was recently pointed out, e.g., in the review paper of Gerberich and collaborators [16]. Indeed, several case studies of metals investigated at the nano- and larger scales by in situ TEM emphasize surface alteration that could influence experimental measurements. A typical example is the case study of Han and collaborators [17] focused on Fe NPs under compression. Fe NPs exhibit a thin $\mathrm{Fe}_{2} \mathrm{O}_{3}$ layer at their surface that could be one of the source of discrepancies on the measured yield stress. Furthermore, MD simulations of oxidized $\mathrm{Al}$ and $\mathrm{Fe}$ NWs [18,19] under tensile deformation demonstrate that surface oxidation enhances ductility of NWs, reduces their yield stress, and lowers the onset of plasticity. Controlling the surface oxidation is challenging both in experiments and MD studies [18]. Indeed, only few interatomic potentials, e.g., ReaxFF, COMB3) allow simultaneous modeling of metals and relative oxide phases [20-22]. However, due to charge variation calculations, these potentials induce high CPU cost, and therefore, they are restricted to relatively small systems (see, e.g., Refs. [18,19]). Furthermore, they are often characterized by a low transferability to model mechanical properties.

Besides the effect of oxidation mentioned above, surface amorphization can also be observed [23,24]. Such an alteration of the surface state is shown to influence mechanical 
properties and dislocation nucleation processes as, for instance, in the case of amorphous-crystalline $(\mathrm{cr}-a)$ core-shell Si nanowires [25,26].

The aim of this study is to investigate the influence of an amorphous surface layer on the mechanical response of metallic NPs using MD simulations. Due to the aforementioned reasons that commonly hamper MD studies of metal-oxide configurations, we focus here on crystalline NPs surrounded by an amorphous layer. In order to streamline the approach, we focus on monoatomic $\mathrm{Ni}$ systems, assuming that the methods used here could apply to other metallic nanocrystals and lead to qualitatively similar results. In particular, this approach allows avoiding complex one-to-one comparison between monoatomic crystalline NPs and $c r$ - $a$ NPs, which have different chemical compositions, e.g., comparing the mechanical response of a $c r \mathrm{Cu} \mathrm{NP}$ with that of a $c r-a \mathrm{Cu} / \mathrm{CuZr} \mathrm{NP}$ ). In the following, we will show how such an approach using monoatomic material can bring significant semiquantitative information about the influence of an amorphized/oxidized surface layer on the mechanics of metallic nano-objects.

Metallic melts can be solidified into a glassy state through rapid quenching down to low temperature. Monoatomic metals are particularly known for their low glass-forming ability that makes the sample elaboration procedure extremely challenging [24]. For monoatomic metallic systems, vitrification can be only achieved with cooling rates in excess of $10^{12} \mathrm{~K} / \mathrm{s}$, using, for instance, such a method as explosive spraying [27] or ultrafast liquid quenching [24], which allow reaching up to $10^{14} \mathrm{~K} / \mathrm{s}$ quench rate. Such elevated quench rates are well adapted for MD calculations typically characterized by a timescale in the nanosecond range. Two recent atomic-scale studies report the structure of amorphous monoatomic $\mathrm{Ni}$ [28] and Al [29]. Also, the conditions for metallic melts vitrification are investigated and analytically described for single-component $\mathrm{Ni}, \mathrm{Al}$, and $\mathrm{Cu}$ systems by Fedorchenko [30]. Among the tested compositions, Ni was shown to have the highest glass formation tendency due to its high melting temperature.

In this work, we aim to design a procedure allowing to build $c r-a$ nanospheres with pure $\mathrm{Ni}$ composition, accounting for the unstable nature of amorphous $\mathrm{Ni}$. Then, $\mathrm{Ni}$ NPs with various thicknesses of amorphous layer are compressed following a well established method designed to mimic TEM/SEM nanocompression tests [12,14,31]. The mechanical response of the particles is investigated and discussed for various amorphous surface layer thicknesses. Particular attention is paid to the onset of plasticity, both in the crystalline core and the amorphous layer of the NPs.

\section{SIMULATION METHODS}

Several embedded-atom-method (EAM) potentials are known to accurately reproduce the structure and the essential mechanical properties of crystalline FCC Ni $(c r-\mathrm{Ni})$, while the information on amorphous $\mathrm{Ni}(a-\mathrm{Ni})$ structure is rather scarce [28]. Here, we examine the transferability of three different EAM potentials to reproduce the structure of monoatomic $\mathrm{Ni}$ glass: (i) Angelo, Moody, and Baskes [32]; (ii) Mishin [33]; and (iii) Mendelev et al. [34]. These potentials are referred hereafter as AMB-95, Mish-04, and Mend-12, respectively.
The simulations are performed using the open-source code LAMMPS [35]. A time step of $2 \mathrm{fs}$ is used in all MD simulations; temperature is controlled using the Nosé-Hoover thermostat [36]. The atomic samples used in MD simulations are designed and manipulated using the command-line program ATOMSK [37]. The atomic structures as well as elementary deformation processes are analyzed using OVITO [38].

\section{A. Creating the amorphous Ni structure}

Following the work of Trady et al. [28], the $a$-Ni bulk structure is obtained by rapid cooling of liquid $\mathrm{Ni}$. The investigated atomic arrays are fully periodic and contain 6912 atoms $(12 \times 12 \times 12$ cubic cell, with the simulation box size of approximately $4 \mathrm{~nm}$ ). In order to obtain the amorphous structure, the relaxed $c r-\mathrm{Ni}$ simulation cell undergoes the following steps: gradual increase of temperature up to $2500 \mathrm{~K}$ $\left(10^{12} \mathrm{~K} / \mathrm{s}\right.$ rate) within the NPT ensemble $\rightarrow$ equilibration of liquid $\mathrm{Ni}$ at $2500 \mathrm{~K}$ using the NVT ensemble for $100 \mathrm{ps} \rightarrow$ fast NPT quench $\left(10^{14} \mathrm{~K} / \mathrm{s}\right)$ of the system down to $10 \mathrm{~K}$, followed by a structural minimization using the conjugate gradient algorithm until the global force on all atoms drops below $10^{-6} \mathrm{eV} / \AA\left(1.602 \times 10^{-15} \mathrm{~N}\right)$. The obtained amorphous and liquid $\mathrm{Ni}$ structures are analyzed using the radial distribution function (RDF) and the bond-angle analysis [39] as implemented in OVITO [38].

\section{B. Building crystalline and crystalline-amorphous NPs}

For purely $c r-\mathrm{Ni}$ systems, $\langle 100\rangle$-oriented nanospheres are carved from a perfect FCC crystal. The $c r-a$ NPs designed for compression are characterized by a total radius $R=10 \mathrm{~nm}$ and a shell thickness $r$ varying from 2 to $6 \mathrm{~nm}$. Additional sample NPs with total radius $R=15$ and $20 \mathrm{~nm}$ are specially built to test the influence of the system size and shell thickness on the crystallization rate. The sample elaboration procedure of $c r-a$ systems is less straightforward than for the cr case. In the literature, one can find different methods for the design of monoatomic $c r-a$ core-shell nanocomposites, e.g., Refs. [26,40-42], which in general can be subdivided into high-temperature and low-temperature approaches (referring to the temperature at the interphase between amorphous and crystalline phases).

The high-temperature method. This strategy requires heating (until melting) and subsequently quenching the outer part of a nanocrystal, while keeping its core region at low temperature [26,42]. Practically, the system is subdivided into two concentric regions (corresponding to core and shell) where two different thermostats are applied. Similarly to the procedure described in Sec. II A, subsequent melting and quenching stages are used here to make the amorphous layers, whereas a constant temperature of $1 \mathrm{~K}$ is set for the core of the NPs.

Previous MD studies of $c r-a$ core-shell Si NWs [26,42] alternatively suggest creating an amorphous surface layer from the random distributions of atoms (with the density of the amorphous phase) annealed above the glass transition temperature, which is commonly of the order of $\frac{1}{2} T_{m}$. While being effective for amorphous $\mathrm{Si}$, this strategy fails here for Ni: the tested Mish-04 and Mend-12 potentials do not yield to a glassy structure from such atomic distribution. 
The low-temperature method. Dealing with pure Ni cr-a systems (where the $a$-phase is unstable), one cannot completely exclude superfluous crystallization of the amorphous surface layer. However, it is possible to slow down this process while decreasing temperature at the $c r$ - $a$ interphase during sample fabrication. Thus, alternatively to the hightemperature methods, a core-shell particle can be created using the low-temperature approach [40,41], i.e., via merging a crystalline FCC core with an amorphous shell, the two parts being carved from the relaxed bulk $c r-\mathrm{Ni}$ and $a-\mathrm{Ni}$, respectively. This method precludes applying high temperatures to the composite system, and therefore inhibits the amorphousto-crystal transformation at the interphase. However, one should bear in mind that the interphase between "merged" phases requires structural optimization. In this work, NPs with an amorphous surface layer are first subjected to energy minimization using conjugate gradient, and then equilibrated for $100 \mathrm{ps}$ at $0.2 \mathrm{~K}$ using the Nosé-Hoover thermostat and free-boundary conditions.

\section{Compression of crystalline-amorphous NPs}

The uniaxial compression of $\langle 100\rangle$-oriented NPs is performed by displacing two infinite (top and bottom) flat force fields along the $z$ axis, similarly to the procedure described in [11,31]. The effective stiffness $K$ of each indenter is set to $1000 \mathrm{eV} / \AA^{3}$. The indenters are displaced towards each other at a constant rate equivalent to engineering strain rate of $10^{8} \mathrm{~s}^{-1}$. In order to hold the crystallization of the $a-\mathrm{Ni}$ as slow as possible, a low temperature of $0.2 \mathrm{~K}$ is set during the whole compression test. During compression, the evolution of the forces $F$ applied by the indenters as well as the contact surface $S$ are systematically monitored and recorded. The contact surface area is computed based on the Delaunay triangulation of the atomic positions combined with a probe sphere approach with a critical radius $R_{\alpha}[43,44]$. In this study, we employ the $R_{\alpha}$ parameter that covers the second coordination sphere in $a-\mathrm{Ni}$ and in $c r-\mathrm{Ni}$ for NPs with and without amorphous shells, respectively. For each recorded deformation step, the true compression stress is then defined as $F / S$.

\section{RESULTS AND DISCUSSION}

\section{A. Characterization of the amorphous Ni structure}

In this section, we describe the $a$ - $\mathrm{Ni}$ bulk structures obtained using the AMB-95, Mish-04, and Mend-12 EAM potentials.

As previously described in Sec. II A, the glassy state of $\mathrm{Ni}$ is reached by rapid cooling of melt with the quenching rate $10^{14} \mathrm{~K} / \mathrm{s}$. Upon the temperature decrease, structural changes in the system are monitored and the local structure evolution is described via the radial distribution function (RDF) $g(r)$ as shown in Fig. 1. At high temperature, the RDF exhibits a typical liquidlike shape [Fig. 1(a)] with no signs of long-range order. By decreasing the temperature, the first peak $R_{1}(2.4 \AA)$ becomes higher and sharper, and the second peak $(4.5 \AA)$ splits into two smaller ones $R_{2}$ and $R_{3}$ located between 4 and $5 \AA$, indicating an increase of the short range order (SRO). The splitting becomes more pronounced with temperature decrease. As it was previously shown in Refs. [28,45], the
TABLE I. Structural properties of monoatomic Ni glass modeled using Mish-04 [33], AMB-95 [32], and Mend-12 [34] EAM potentials.

\begin{tabular}{lcccccr}
\hline \hline Potential & $E_{a} / E_{c r}$ & $V_{a}\left(\mathrm{~nm}^{3}\right)$ & $V_{a} / V_{c r}$ & $\rho_{a}\left(\mathrm{~g} / \mathrm{cm}^{3}\right)$ & $\rho_{a} / \rho_{c r}$ & $T_{g}(\mathrm{~K})$ \\
\hline Mish-04 & 1.027 & 77.202 & 1.025 & 8.726 & 0.945 & 1050 \\
AMB-95 & 1.025 & 79.740 & 1.058 & 8.448 & 0.976 & 750 \\
Mend-12 & 1.031 & 77.508 & 1.030 & 8.691 & 0.971 & 600 \\
\hline \hline
\end{tabular}

third peak $R_{3}$ occurs due to increasing number of icosahedral clusters, while the second peak $R_{2}$ can be rather explained by the presence of 13- and 14-fold coordination polyhedra like $\langle 0,1,10,2\rangle$ and $\langle 0,2,8,4\rangle$.

From the obtained evolution of the RDF with temperature, one can define the glass transition temperature $T_{g}$ based on the Wendt-Abraham parameter $R^{\mathrm{WA}}=g_{\min } / g_{\max }$ where $g_{\min }$ and $g_{\max }$ are the first minimum and maximum of the $g(r)$ curve. Among the three tested EAM potentials, the Mish04 potential provides the highest $T_{g}=1050 \mathrm{~K}$ [Fig. 1(a)], close to $60 \%$ of the melting temperature $T_{m}=1701 \mathrm{~K}$ (as provided in Ref. [34]). Transition temperatures $T_{g}$ computed with AMB-95 and Mend-12 potentials (Table I) are $750 \mathrm{~K}$ and $600 \mathrm{~K}$, respectively, which is rather close to $\frac{1}{3} T_{m}$.

Figure 1(b) compares the RDF of the relaxed Ni glass structures computed with the three different EAM potentials and the experimental RDF curve characterizing $a$ - $\mathrm{Ni}$ [46]. Overall, the shape of the calculated RDFs is in agreement with experimental data. The apparent difference in the heights of the first peak $R_{1}$ between modeled and experimental $g(r)$ could be associated to higher temperature conditions in the experiment, as previously discussed in Ref. [45]. All the functions obtained from MD simulations exhibit characteristic splitting of the $R_{2}$ and $R_{3}$ peaks, typical for the glassy state of $\mathrm{Ni}$ [45]. Although the splitting of the experimental curve is less prominent than that obtained from EAM potentials, the positions of $R_{2}$ and $R_{3}$ peaks of all the curves correlate well with each other. The $g(r)$ curve of the AMB-95 potential has the highest $R_{1}$ peak and the most prominent splitting of the $R_{2}$ and $R_{3}$ peaks, suggesting the highest $\mathrm{SRO}$ among the tested potentials. On the other hand, the splitting of the peaks provided by the Mend-12 potential appears to be the weakest indicating the structure with lower SRO. For the Mish-04 curve, the second, fourth, and fifth RDF peaks are slightly shifted towards lower distances, which results in a denser $a$-Ni structure with relatively smaller volume compared to those obtained with the two other potentials (see $\rho_{a}$ and $V_{a}$ values in Table I)

Table I summarizes the computed properties of the $a-\mathrm{Ni}$ structure. As mentioned above, Mish-04 potential provides the highest absolute value $\rho_{a}$ among the tested potentials; however, this glass structure is $5.5 \%$ less dense than crystalline FCC Ni (see $\rho_{a} / \rho_{c r}$ values), while for the other potentials the difference is only $2.4 \%-2.9 \%$. All the tested potentials yield a potential energy of the glassy structures of the order of $2.5 \%-3 \%$ higher than that of FCC Ni (described via $E_{a} / E_{c r}$ in Table I).

Based solely on the analysis of the $a$-Ni glass structures (Fig. 1, Table I), one cannot determine which interatomic 

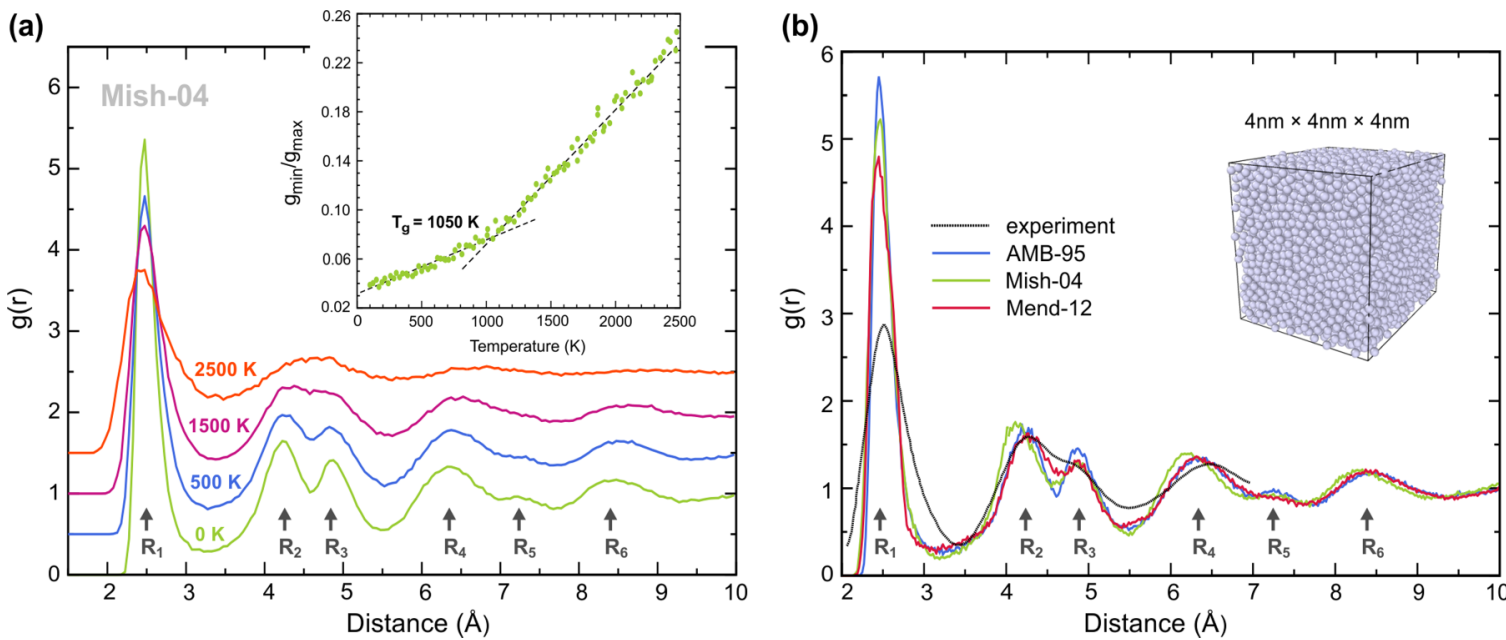

FIG. 1. (a) Evolution of the RDF with temperature during quenching of liquid Ni computed using the Mish-04 potential. The inset illustrates calculations of the glass transition temperature $T_{g}$ based on the Wendt-Abraham parameter $R^{\mathrm{WA}}=g_{\min } / g_{\max }$. (b) Comparison of the RDFs of the relaxed $a$-Ni structures obtained with the AMB-95, Mish-04 and Mend-12 interatomic potentials with the experimental RDF of $a$-Ni [46].

potential is better suited for modeling $c r-a$ Ni systems. Thus, in the next section, we will further test the transferability of all the three EAM potentials to model composite $c r-a$ Ni NPs.

\section{B. Crystalline-amorphous core-shell NPs}

Here we investigate the transferability of the three EAM potentials for modeling $c r-a$ core-shell systems. In particular, we examine the effects of temperature, shell thickness and compressive stress on the stability of $c r-a$ Ni core-shell NPs. Considering a composite system where a metastable $(a-\mathrm{Ni})$ and stable $(c r-\mathrm{Ni})$ phases coexist, we cannot completely exclude partial crystallization of the amorphous shell. Therefore we intend to find a combination of factors acting together (namely, the sample elaboration method, interatomic potential, temperature and shell thickness) that allow preserving the $a$-Ni shell under compression, at least until the onset of a first plastic event in the $c r$ core. In order to compute the crystallization rate, we monitor the position of the $c r$ - $a$ interface along the six $\langle 100\rangle$ directions, which correspond to the fastest crystallization directions in our case, as a function of time.

\section{Fabrication of core-shell NPS}

Obtaining a noncrystalline shell directly on a FCC substrate using the high-temperature approach (from melt) requires to impose local temperatures larger than $T_{m}$ and subsequently perform a rapid quench (see Secs. II A and II B). The initially introduced high temperature at the core-shell interface leads to a very fast crystallization $(>1 \AA / p s)$ of the shell during quenching. Moreover, due to the extremely high crystallization rate, the newly crystallized FCC domain contains a large amount of defects such as stacking faults (SFs) [Fig. 2(a)].

Previous MD studies of $c r-a$ core-shell Si NWs [26,42] also point out this drawback of the high-temperature method resulting in significant uncontrolled recrystallization of the shell and a redundant formation of defects. Here, the uncontrolled change in FCC volume fraction during quenching hinders the one-to-one systematic comparison of the samples, e.g., for mechanical properties. Therefore, for the current study, we will not consider this sample fabrication approach.

In contrast to the high-temperature method described above, the low-temperature approach [40,41], creating an amorphous-crystalline composite via merging the two phases, leads to a decent loss of amorphous structure during sample elaboration without superficial formation of defects [Fig. 2(b)]. Thus the low-temperature method is better suited for the fabrication of the $c r-a$ Ni NPs, and therefore, will be preferred in the rest of the study. The crystallization rate of the amorphous layer during the low-temperature process for the three EAM potentials is further investigated in the next section.

\section{Crystallization rate at low temperature}

In this section, the crystallization rate is studied for a spherical NP with a total radius $R=10 \mathrm{~nm}$ and a shell thickness of $r=2 \mathrm{~nm}(r / R=0.2)$ as a reference system. Table II
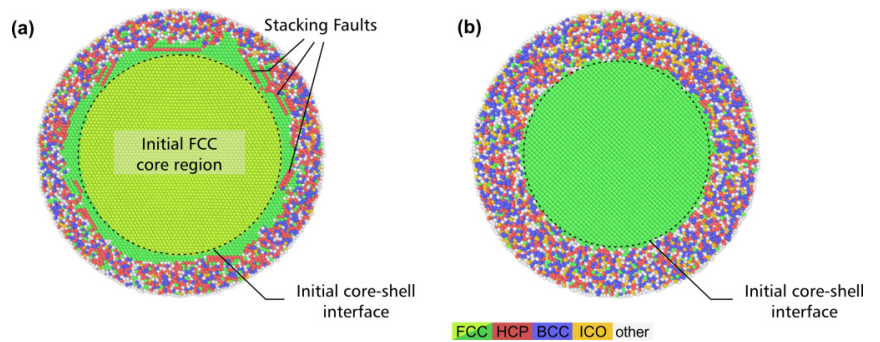

FIG. 2. Core-shell Ni NPs designed using (a) the hightemperature method, i.e., by melting and rapidly quenching the outer part of the particle, and (b) the low-temperature approach via merging crystalline FCC core with amorphous shell. Black dashedlines delimit FCC structures before and after equilibration. The atoms are colored according to their structural type as defined by the bond-angle analysis: FCC (face-centered cubic), HCP (hexagonal close-packed), BCC (body-centered cubic), and ICO (icosahedral). 
(a)

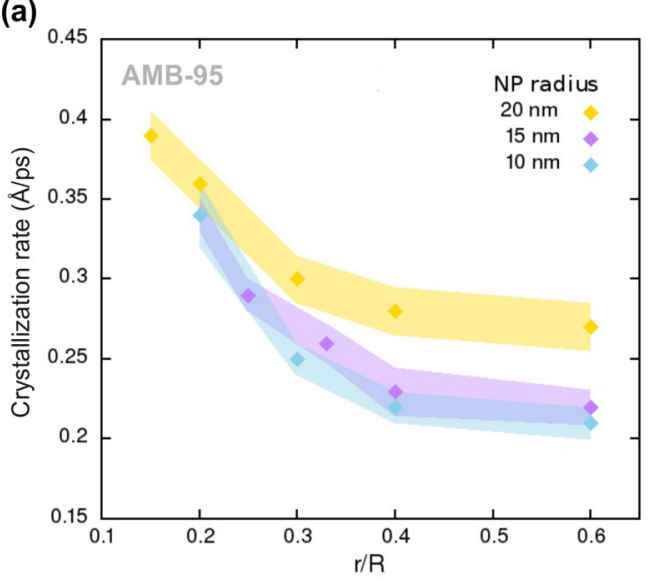

(b)

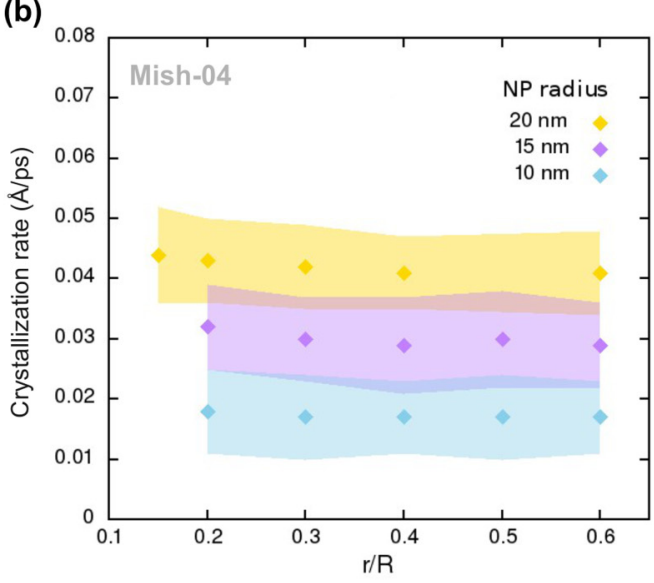

FIG. 3. Crystallization rate of the $a$-Ni layer as a function of the $r / R$ ratio between the thickness of the amorphous shell and the total radius of NPs. The results are presented for (a) AMB-95 [32] and (b) Mish-04 [33] EAM potentials. The error bars result from the difference in crystallization rates obtained for the six directions of $\langle 100\rangle$ axis.

reports crystallization rates deduced from annealing of the NP at $0.2,1$, and $10 \mathrm{~K}$ using the three tested EAM potentials. Among the three tested potentials, Mend-12 leads to the fastest crystallization rate. Even at extremely low temperature (i.e., $T=0.2 \mathrm{~K}$ ), the crystallization rate is approximately $0.53 \AA /$ ps meaning that, even without compressive stress, a $2 \mathrm{~nm}$ amorphous shell will completely crystallize after $40 \mathrm{ps,}$ which is less than the typical timescale for MD compression simulations. Better results are obtained for both the AMB-95 and the Mishin-04 parametrizations with lower crystallization rates (see Table II). Indeed, the Mish-04 potential exhibits crystallization rates ranging from $0.02 \AA / \mathrm{ps}(T=0.2 \mathrm{~K})$ up to $0.09 \AA / \mathrm{ps}(T=10 \mathrm{~K})$, which are one to two orders of magnitude lower than those obtained with Mend-12. Thus, the Mend-12 potential appears to be the least suitable for modeling Ni core-shell NPs. Therefore this potential will not be further investigated in the rest of this study.

\section{Influence of the sample size and shell thickness on the crystallization rate}

Here we examine the effect of the sample geometry on the crystallization rate relying on the Mish-04 and AMB-95 interatomic potentials. Figure 3 illustrates the crystallization rates computed for 20,30 , and $40 \mathrm{~nm}$ diameter nanospheres as a function of the $r / R$ ratio. On this figure, the error bars result from the difference in crystallization rates obtained for the six $\langle 100\rangle$ directions. For both potentials, the crystallization

TABLE II. Influence of the temperature on the crystallization rate (in $\AA / p s$ ) computed for a $c r-a$ core-shell $\mathrm{Ni} \mathrm{NP}$ of $10 \mathrm{~nm}$ radius with $2 \mathrm{~nm}$ amorphous shell using the AMB-95 [32], Mish-04 [33], and Mend-12 [34] EAM potentials.

\begin{tabular}{lccc}
\hline \hline Potential & $0.2 \mathrm{~K}$ & $1 \mathrm{~K}$ & $10 \mathrm{~K}$ \\
\hline Mish-04 & 0.02 & 0.06 & 0.09 \\
AMB-95 & 0.18 & 0.46 & 0.80 \\
Mend-12 & 0.53 & 1.22 & 2.28 \\
\hline \hline
\end{tabular}

rate increases with the size of the NP. This tendency can be explained by the relative increasing amount of FCC crystal in the sample. For the AMB-95 potential, characterized by a faster crystallization rate [Fig. 3(a)], we find an apparent dependence on the $r / R$ ratio such as the crystallization rate increases as the relative volume of amorphous phase in a sample decreases ( $r / R$ ratio decreases). For all the examined NP sizes, the crystallization rate saturates when the shell thickness reaches $40 \%$ of the total sample radius. The crystallization rate obtained with Mish-04 remains rather constant for a given NP size regardless of the shell thickness [Fig. 3(b)]. Here, the crystallization rate appears to be less sensitive to the relative volume ratio between amorphous and crystalline phases. In this case, due to the very low transformation rate, the actual increase of the transformation rate with decreasing fraction of amorphous phase can be hidden within the error bar.

\section{Influence of stress on the crystallization rate}

In the previous Secs. III B 1-III B 3, we have shown that for the investigated $c r-a$ Ni NPs, a progressive crystallization of the amorphous surface layer is ubiquitous and unpreventable. Although it was possible to identify the optimal conditions inhibiting this process, e.g., low temperature and suitable EAM potential), the crystallization rate is likely to be further enhanced by the applied stress during compression. In order to quantify the evolution of the crystallization rate under stress, we further investigate the $c r$ - $a$ Ni NPs with $r=2 \mathrm{~nm}$, $R=10 \mathrm{~nm}$ under compression at $0.2 \mathrm{~K}$ using the AMB-95 and Mish-04 potentials following the protocol described in Sec. II C. Figure 4 brings together the positions of the top indenter and the $c r-a$ interphase during compression tests performed at $10^{9} \mathrm{~s}^{-1}$ engineering strain rate. The compression stress promotes the increase of the crystallization rate from 0.18 to $0.47 \AA /$ ps and from 0.02 to $0.07 \AA /$ ps for the AMB-95 and the Mish-04 potentials, respectively. With the AMB-95 potential, the amorphous surface layer quickly crystallizes along the compression direction at approximately $6 \%$ strain, where the recrystallized $c r-\mathrm{Ni}$ comes in contact with the indenter. This process is delayed with the Mish-04 interatomic 


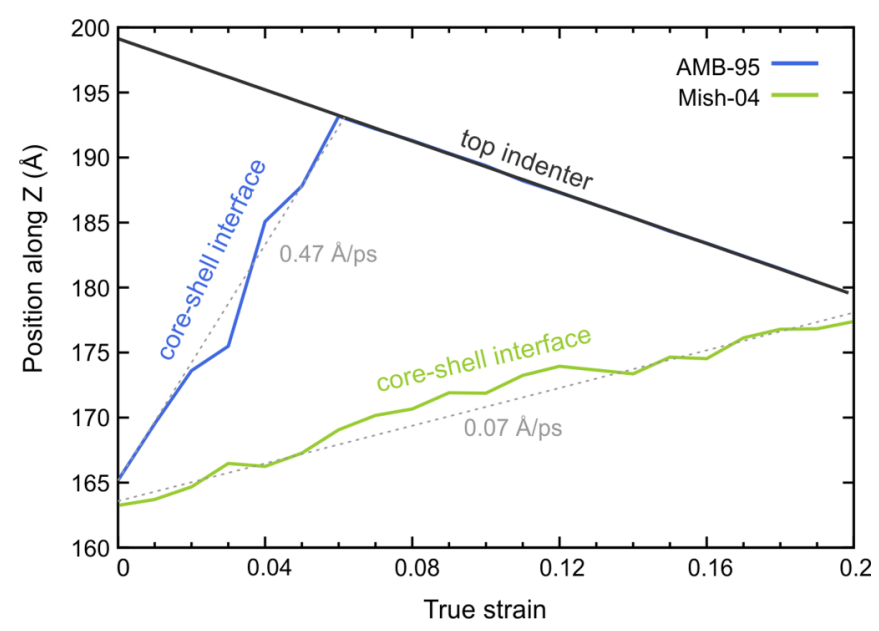

FIG. 4. Crystallization rate of $a$-Ni surface layers for $R=10 \mathrm{~nm}$ and $r=2 \mathrm{~nm}$ as derived from the propagation of the $c r$ - $a$ interphase during compression at $10^{9} \mathrm{~s}^{-1}$ strain rate and $T=0.2 \mathrm{~K}$.

potential, which allows up to $20 \%$ strain without complete crystallization of the amorphous surface layer.

Based on these results, we conclude that, among the tested EAM potentials, only Mish-04 can allow investigating $c r-a$ Ni NPs under compression. This interatomic potential will be further used in the last section of the study that focuses on the compression of Ni-based NPs.

\section{Nanocompression simulations}

\section{Mechanical response}

In this section, we perform MD nanocompression tests of purely $c r$ and $a$-Ni NPs with a total radius $R=10 \mathrm{~nm}$, as well as of the $c r-a$ systems of same size with a shell thickness $r$ varying from 2 to $6 \mathrm{~nm}(r / R$ ratio ranging from 0.2 to 0.6$)$. The calculations are performed at constant compression rate of $10^{8} \mathrm{~s}^{-1}$ relying on the Mish-04 interatomic potential which provides the slowest crystallization rate during the sample fabrication [Table II, Fig. 3(b)] and compression processes [Fig. 4].

The computed true compressive stress versus true strain curves are shown in Fig. 5 where one can distinguish three different sample cases depending on the $r / R$ ratio. First, the typical mechanical response of the $c r$-Ni NP is similar to that in the previously published compression tests of FCC metallic nanospheres, e.g., Refs. [11,47]). It is initially characterized by an elastic regime up to 5.3\% strain and a yield stress of 13.5 GPa. After yielding, the curve shows stress drops due to successive surface dislocation nucleation (SDN) events.

While the variations of the $c r$-Ni NP curve are singular and characterized by ups and downs at particularly high stress, the stress-strain curves of $c r$ - $a$ NPs with $r / R$ ranging from 0.3 to 0.6 as well as the one of the $a$ $\mathrm{Ni}$ NP are significantly different and exhibit the second type of behavior. These systems are characterized by a lower Young's modulus, which is typical of amorphous materials, and a softer elastoplastic transition, as also observed in the case of core-shell NWs under tension $[18,19]$, and more generally speaking, by a smoother mechanical

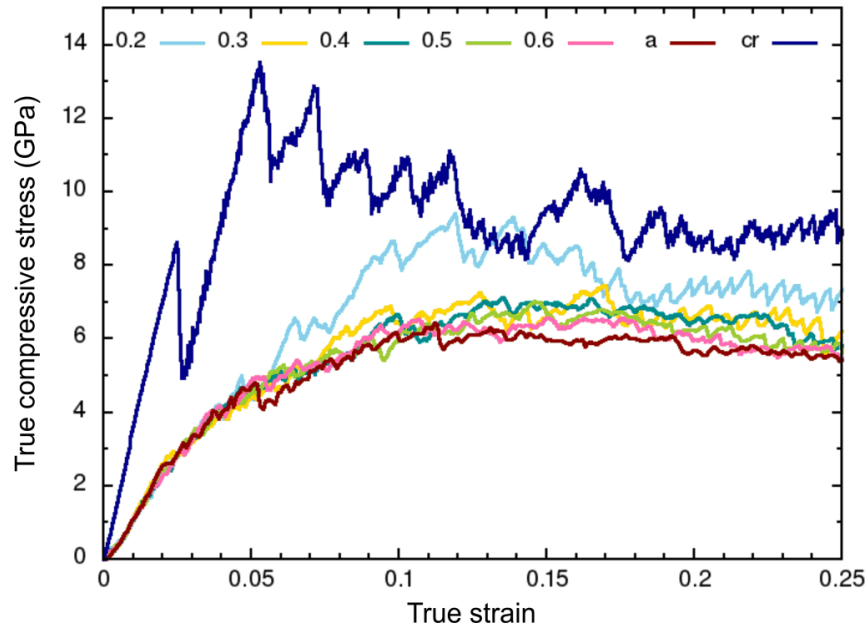

FIG. 5. Stress-strain curves computed for purely crystalline ( $\mathrm{cr}$ ), amorphous $(a)$, and $c r-a$ Ni NPs. Numbers in the legend correspond to the ratio $r / R$ between the amorphous surface layer thickness $r$ and the total radius $R=10 \mathrm{~nm}$ of the NPs.

response, i.e., lower stress range and variations, when compared to the $c r$-Ni sample.

Third, the $r / R=0.2$ sample behaves in between the $c r-\mathrm{Ni}$ and the $a$-Ni data sets, i.e., the $r / R=0.2$ stress-strain curve exhibits an elastic regime that mimics to the one of the $a-\mathrm{Ni}$ $\mathrm{NP}$ up to $\epsilon=5.9 \%$. Then, it diverges and increases up to the $c r$-Ni response. This behavior occurs due to the particularly fast shell crystallization, which ends at $\epsilon=5.9 \%$ when the FCC Ni crystal comes in contact with the indenters. At larger strains, the stress decreases and a steady state of about $7.2 \mathrm{GPa}$ takes place.

\section{Elementary deformation processes}

$\mathrm{Cr}$-Ni NPs. In Fig. 5, the stress drop at $\epsilon=2.5 \%$ on the $c r$-Ni NP stress-strain curve results from the surface accommodation of two unstable, "hillock" [11] pyramidal dislocation structures (SFs bounded by four $\frac{1}{3}\langle 100\rangle$ edge dislocations), typical of simulated FCC nanospheres when compressed along $\langle 001\rangle$ [Fig. 6(a)]. This process does not comply with the Hertz model, which assumes subsurface maximum stress concentration in the case of perfect sphere under compression (see, e.g., Ref. [48]). Here, one can assume that homogeneous dislocation nucleation is not favoured (when compared to SDN) due to the competition between subsurface shear stress concentration on one hand, and nanoscale properties such as surface atomic discretization, orientation of surface ledge, and dislocation line energy on the other hand. Nevertheless, while this process recently drew particular attention $[11,47,49]$, it might not be realistic. Indeed, the pyramidal dislocation structure, which, up to now, has only been observed in MD simulations, results from the interaction of four Shockley partial dislocations simultaneously nucleated in four $\frac{1}{6}\langle 112\rangle\{111\}$ slip systems of maximum Schmid factor.

The coincidence of the four nucleation events is a necessary condition to ensure the pyramidal shape of the dislocation reaction, and such a symmetric structure can only be formed due to three reasons incident to the simulation. 


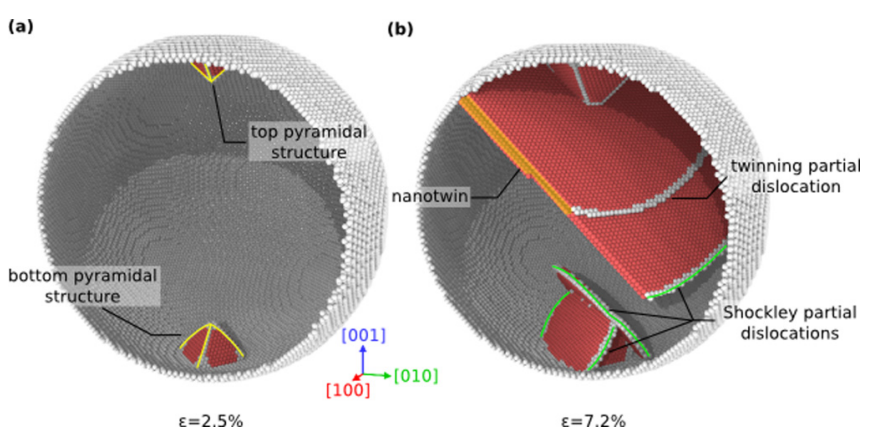

FIG. 6. Surface dislocation nucleation $(\mathrm{SDN})$ in a $20 \mathrm{~nm} \mathrm{cr}$ Ni NP. (a) The pyramidal dislocation structure that occurs at the early stage of plastic deformation $(\epsilon=2.5 \%)$. The yellow curves correspond to $\frac{1}{3}\langle 100\rangle$ edge dislocation lines. (b) Multiple SDN events and nanotwin growth at $\epsilon=7.2 \%$. Shockley partial dislocations are shown in green; the nanotwin is depicted by an orange area. The atoms are colored according to the structural type defined by the bond-angle analysis: HCP is shown in red (SFs and twin boundaries), defective regions are white (surfaces, dislocation cores, disconnections). For the sake of clarity, FCC atoms are removed.

First, the indenter and the sample should be perfectly aligned in the simulation along the [001] direction, which ensures the same Schmid factor of 0.408 in the $\frac{1}{6}[1 \overline{1} 2](1 \overline{1} \overline{1})$, $\frac{1}{6}[\overline{1} \overline{1} 2](111), \frac{1}{6}[1 \overline{1}](\overline{1} 1 \overline{1})$, and $\frac{1}{6}[112](\overline{1} \overline{1} 1)$ slip systems. A change of a fraction of degree would break the symmetry of the Schmid factors and, consequently, the simultaneousness of the four events. Such perfect alignment cannot be currently reached in experiments, especially in in situ TEM nanocompression tests where setup geometry optimization is one of the major research objectives [12,50,51]. The second reason arises from the actual shape of the NPs. Here, we modeled perfectly spherical $\langle 100\rangle c r-N i$ NPs. These NPs are highly symmetric and all their six $\langle 100\rangle$ axes are strictly identical in terms of geometry and number of atoms building $\{100\}$ surface layers. This perfect geometry also contributes to the coincidence of SDN events responsible for the pyramidal dislocation structure. In experiments, such a high level of "perfection" in terms of NP shape cannot be achieved. Finally, the third reason originates from the thermally-activated nature of SDN, which is assumed to only happen at extremely high stress in MD calculations due to the high compression rate and small timescale (of the order of ns) conditions. In experiments, the timescale is larger hence delaying probabilistic temperature-dependent events in terms of stress and time. Again, this factor would reduce the possibility of simultaneous SDN and the pyramidal structure to occur.

While the formation of the pyramidal structure might influence the early plastic deformation regime, its mechanical instability (it fails at $\epsilon=5.3 \%$ ) allows the system to further deform by more conventional deformation processes such as uncorrelated Shockley partial dislocation and nanotwins nucleation. Indeed, the stress drops observed on the purely $\mathrm{cr}$ NP response at $\epsilon=5.3 \%$ and $7.3 \%$ refer to the multiplication of several partial dislocations, from the top and bottom of the pyramidal dislocation structures, which quickly leads to the formation of extended SFs and deformation nanotwins
[Fig. 6(b)]. At larger strains, stress variations are characterized by subsequent SDN events.

Cr-a Ni NPs. Due to the presence of the amorphous surface layer, the deformation processes and mechanical response of $c r-a$ Ni NPs are strongly different from their purely crystalline counterparts. Here, we first focus on the $r / R=0.3$ sample, which is considered as a proxy for $c r-a \mathrm{Ni}$ samples with $r / R \geqslant$ 0.3 . Then, the deformation of the $r / R=0.2$ sample will be described.

In contrast to the $c r$-NPs, one cannot clearly distinguish the elastic regime from the plastic deformation domain in the $c r-a$ and $a$-Ni systems at small strains (Fig. 5). This suggests a complex deformation process where small irreversible plastic events already occur in the linear elastic regime, followed by a smooth transition into a more effective plastic regime. Such behavior is commonly observed in amorphous systems [52-54]. This interpretation is also confirmed by the analysis of the nonaffine squared atomic displacements describing the distribution of local plastic shear in the compressed sample. Figure 7(a) clearly illustrates that the $a$ layer is sheared before the $c r$ core. This effect is particularly prominent in the areas close to the top and bottom virtual indenters, where the accumulated shear is maximum. Even if located close to the contact with the indenter, the plastic shear in the $a$-shell is rather homogeneous and no shear bands are observed during the whole deformation process. The absence of shear bands can be explained by the dimension of the amorphous surface layer, which is too thin to promote shear banding $[55,56]$ as well as by the low relaxation state of the glassy structure (the $a-\mathrm{Ni}$ was obtained with extremely high quenching rates). Indeed, unrelaxed glasses rather deform by homogeneous deformation when compared to the relaxed amorphous structures (see, e.g., Ref. [57]). A similar homogeneous process is observed in the case of the purely $a$-Ni NP.

For the $r / R=0.3 \mathrm{NP}$, the first plastic event in the $c r$ core occurs due to the nucleation of $\frac{1}{6}[1 \overline{1} \overline{2}](\overline{1} 1 \overline{1})$ partial dislocation from the $c r-a$ interface at $\epsilon=13.6 \%$ [Fig. 7(b)]. In this case, the interface dislocation nucleation (IDN) site is not located in the center of the compression contact area like it was for the purely $c r-\mathrm{Ni}$ NP. Instead, it occurs aside, where the $c r-a$ interface is also highly sheared [see Fig. 7(b)]. This behavior confirms both the role of the atomic local environment at the interphase as well as the transmission of plastic shear from the $a$ phase to the $c r$ phase by the IDN process, as already observed in other $c r-a$ composites, such as nanolaminates [58]. At the same time, the complementary process, i.e., the transmission of plastic shear from the $c r$ to the $a$ phase, is also observed once the Shockley dislocation reaches the interface at the opposite side of the NP and grows further as a nanotwin [Fig. 7(c)]. Dislocation plasticity in the NP core occurs at $\epsilon=$ $13.6 \%$, much larger than that in the $c r$-NP $(\epsilon=2.5 \%-7.2 \%$ corresponding to the formation and subsequent destruction of the pyramidal structure). This confirms the crucial role of the amorphous surface layer to accommodate both elastic and plastic strains. Thus no pyramidal dislocation structure occurs in the $c r$ - $a$ Ni NPs, even when being compressed along the [001] direction similarly to the $c r-\mathrm{Ni}$ NPs. As such, we assume that, in both simulations and experiments, a thin oxide or amorphous layer at the surface of metallic NPs should 


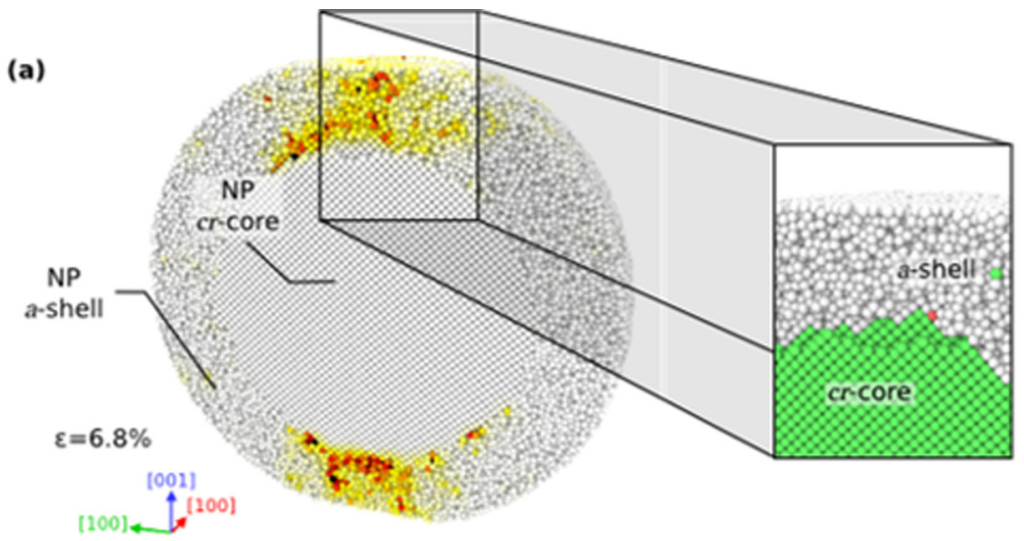

(b)

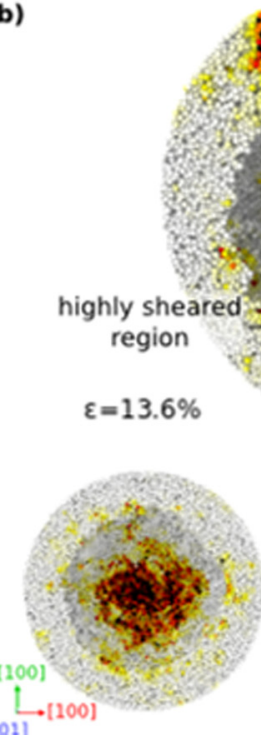

(c)

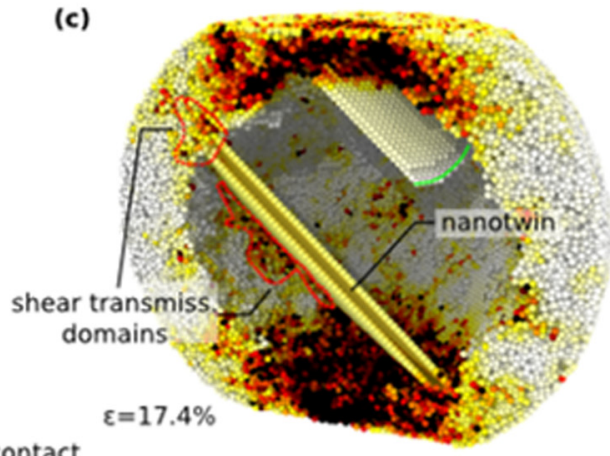

compression contact

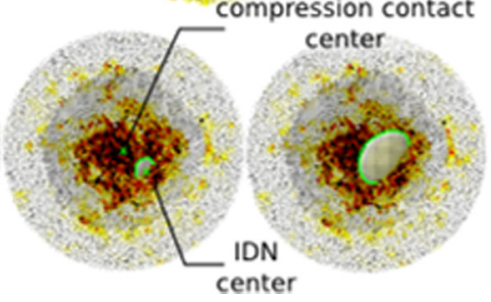

Nonaffine Squared Displacement

(100

FIG. 7. Interface dislocation nucleation (IDN) in the $r / R=0.3 c r-a$ NP. Atoms are colored according to the magnitude of their nonaffine squared displacements [except for the inset (a), where colors refer to crystalline structures: FCC is shown in green, glassy structure in white]. Dashed red and solid green curves refer to specific interfacial regions and partial dislocations, respectively.

prevent the nucleation of the highly symmetric pyramidal dislocation structure at the onset of plasticity.

At larger strains applied to the $c r-a \mathrm{Ni} \mathrm{NP}$, the nanotwin [depicted in Fig. 7(c)] continues to grow, and new dislocations (both partial and perfect) nucleate from the interface in several slip systems. Dislocations and twins interact in a complex manner. This process will be further detailed below. Overall, a similar combination of twinning and dislocation processes is observed in $0.3 \leqslant r / R \leqslant 0.6 \mathrm{cr}-a \mathrm{Ni}$ NPs as expected from the stress-strain curves presented in Fig. 5.

In Fig. 5 , the $r / R=0.2$ stress-strain curve exhibits a higher stress response compared to the other $c r-a$ NPs at strains ranging from $\epsilon=6.5 \%$ to $16.9 \%$. This behavior results from the stress-induced crystallization process of the amorphous surface layer, close to the indenters, as shown in Fig. 8(a). At $\epsilon=7.0 \%$, the top $a$-Ni region is fully crystalized, and the FCC phase comes in direct contact with the indenter. Similar crystallization of the shell occurs in the bottom region at $\epsilon=7.8 \%$, thus bringing both indenters in contact with the cr-Ni core [Fig. 8(a)].

Two major plastic events take place in the $\mathrm{cr}$ core of the NP with the $r / R=0.2$ at $\epsilon=10.1 \%$ and $11.0 \%$. In both cases, the onset of dislocation nucleation occurs at the triple line between the core-shell interface and the indenter [see Fig. 8(b)]. At $\epsilon=10.1 \%$, a leading partial dislocation with Burgers vector $\frac{1}{6}[1 \overline{1} 2]$, quickly followed by a $\frac{1}{6}[211]$

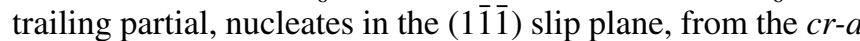
interface, just below the top indenter. Nevertheless, the $\frac{1}{2}[101]$ dislocation rapidly escapes the sample (from the top region) by cross-slip, after a quick interaction with a $\frac{1}{6}[1 \overline{1} \overline{2}](\overline{1} 1 \overline{1})$ dislocation. At $\epsilon=11.0 \%$, one more (11̄i) Shockley partial nucleates from the bottom $c r-a$ interface region, propagates further and crosses the entire sample. This event is followed by the nucleation of a twinning partial dislocation at $\epsilon=12.2 \%$, on the top of the extended SF, leading to the formation of a first nanotwin (NTw1). Furthermore, a $\frac{1}{6}[1 \overline{1} \overline{2}](\overline{1} 1 \overline{1})$ partial dislocation nucleates from the top of the NP, as shown in Fig. 8(b). The deformation of the $r / R=0.2$ sample is particularly interesting as it is merely based on nanotwin interaction processes, which are rarely detailed in the literature. From $\epsilon=12.2 \%$ to $13.3 \%$, the NTw1 grows and the $\frac{1}{6}[1 \overline{1} \overline{2}](\overline{1} 1 \overline{1})$ dislocation shown in Fig. 8 (b) also becomes a nanotwin (NTw2) due to the IDN of a twinning partial dislocation. This process is depicted in Fig. 8(c). At this 

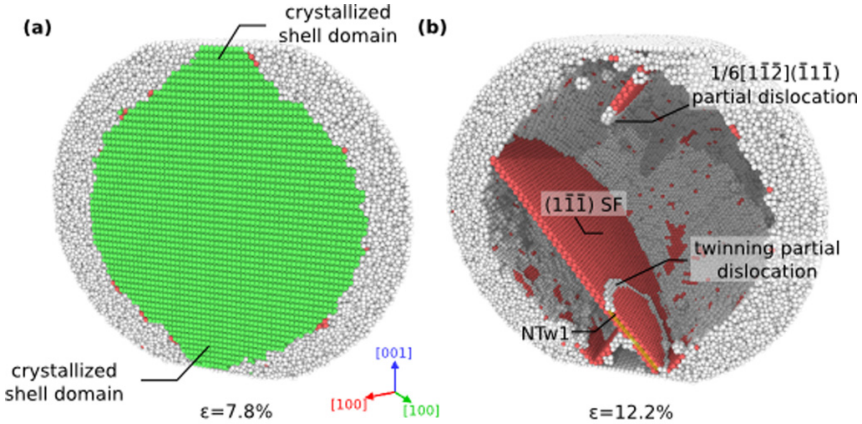

(c)

(d)
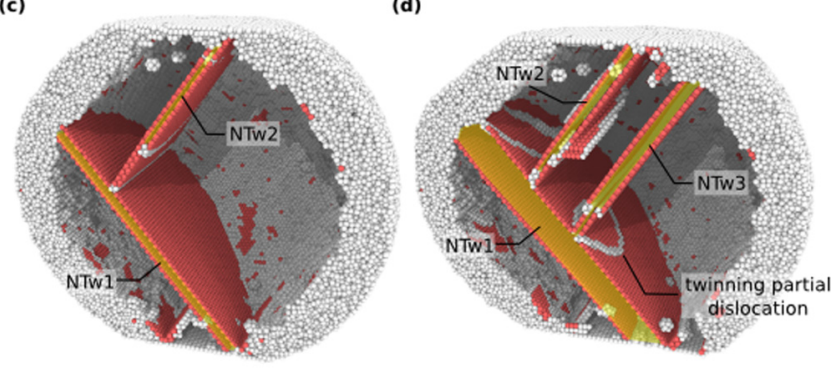

$\varepsilon=13.3 \%$

$\varepsilon=19.4 \%$

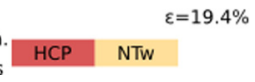

FIG. 8. Deformation processes in $a$-cr core-shell Ni NP with $r / R=0.2$. (a) Crystallization of the shell in the top and bottom regions in contact with indenters. (b)-(d) Nanotwin nucleation, growth and interactions. Three nanotwins refered as NTw1, NTw2, and NTw3, are depicted. The atoms are colored according to the structural type. FCC atoms in (b)-(d) are removed for clarity.

stage, NTw1 behaves as an obstacle to the propagation of the NTw2 and its former partial dislocation. At larger strains, NTw1 continues to grow further while NTw2, as well as an additional nanotwin (NTw3) nucleated in the (111 $)$ plane, are progressively repealed towards the peripheral area of the NP [Fig. 8(d)]. At $\epsilon=19.4 \%$ [Fig. 8(d)], there are two interesting points to address: (i) NTw2 and NTw3 are particularly thin compared to the thicker NTw1. One can observe that as NTw1 fully crosses the whole crystal, it grows much faster than NTw2 and NTw3; (ii) in addition to SDN and IDN, here we show that the emission of twinning partial dislocations can also happen from the twin reaction regions (i.e., at the intercept between two NTw), as illustrated in Fig. 8(d).

\section{Influence of the amorphous surface layer on the mechanical response of $\mathrm{Ni} \mathrm{NPS}$}

From Fig. 5, one can see that the amorphous surface layer strongly affects the mechanical response of metallic NPs under compression. The origins of the decrease in terms of critical yield and flow stresses, for $c r-a$ and $a$ systems as compared to their crystalline counterpart, can be derived from Fig. 9 illustrating the evolution of force and the contact surface area during compression.

At large strains, the contact surface area of $a$ and $c r-a$ systems increases faster than that of the $c r$-Ni NP [Fig. 9(b)]. Such behavior partially explains the relatively low flow stress values of the composite and amorphous NPs compared to the fully crystalline counterpart. It is interesting to note that the surface areas of the core-shell and amorphous particles evolve similarly up to $\epsilon=10 \%$ (corresponding to the $10 \AA$ displacement). Below $\epsilon=8 \%$, the contact areas are in the same range for $c r, c r-a$, and $a$ NPs. However, there are two critical differences between the curves. Firstly, the initial value of the surface area of a partially (or fully) amorphous NP is approximately $8 \mathrm{~nm}^{2}$, while that of the $c r$ nanosphere is closer to $20 \mathrm{~nm}^{2}$. This difference results from the direct contact of the flat crystallographic (001) atomic layers of $\mathrm{cr}$ NPs with indenter, while the rounded surfaces of the relatively disordered amorphous structure produce much smaller contact area at the beginning of the compression test. Secondly, the variation of the contact area is a stepped process in the case of the $\mathrm{cr}$ sample while it is more continuous for $c r-a$ and $a$-NPs. The stepped variation of the contact area curve of the $\langle 001\rangle$-oriented $c r$-NP was recently discussed in the literature and attributed to the interplanar indentation of (001) layers [14,47]. This process does not apply for $c r-a$ NPs as the amorphous overlayer homogeneously reorganizes during the compression, which ensures a smoother evolution of the contact surface. Figure 10(b) emphasizes how the amorphous (a)

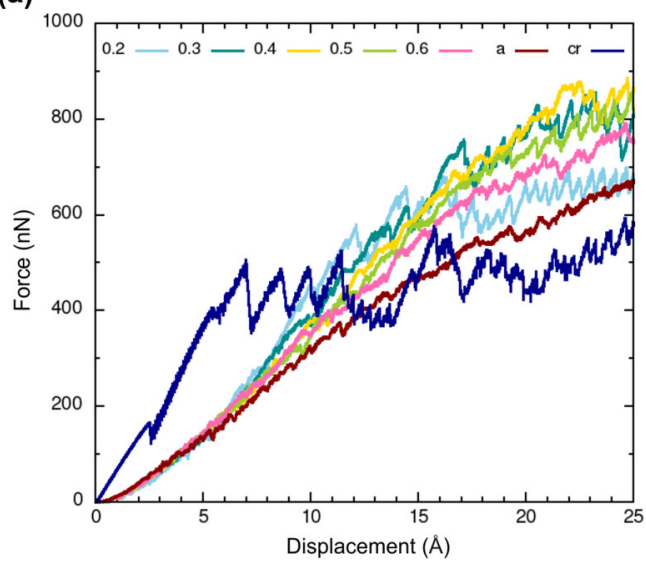

(b)

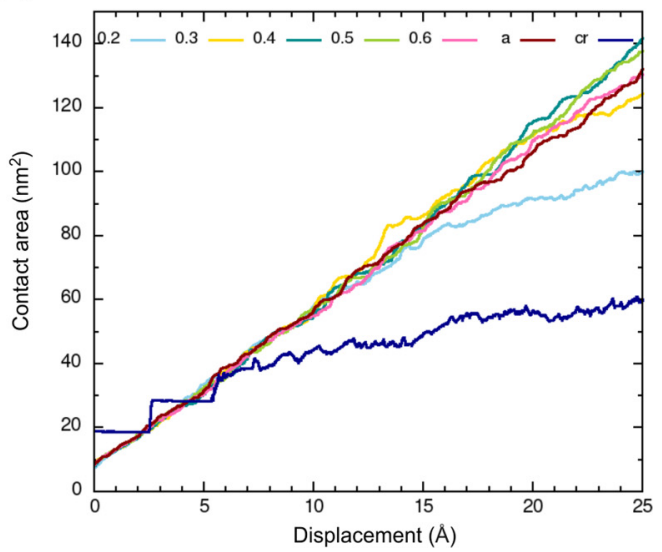

FIG. 9. (a) Force-displacement and (b) contact area-displacement curves. Numbers in the caption refer to the ratio $r / R$ between the amorphous surface layer thickness $r$ and the total radius $R=10 \mathrm{~nm}$ of the NPs; $c r$ and $a$ stand for purely crystalline and purely amorphous NPs respectively. 


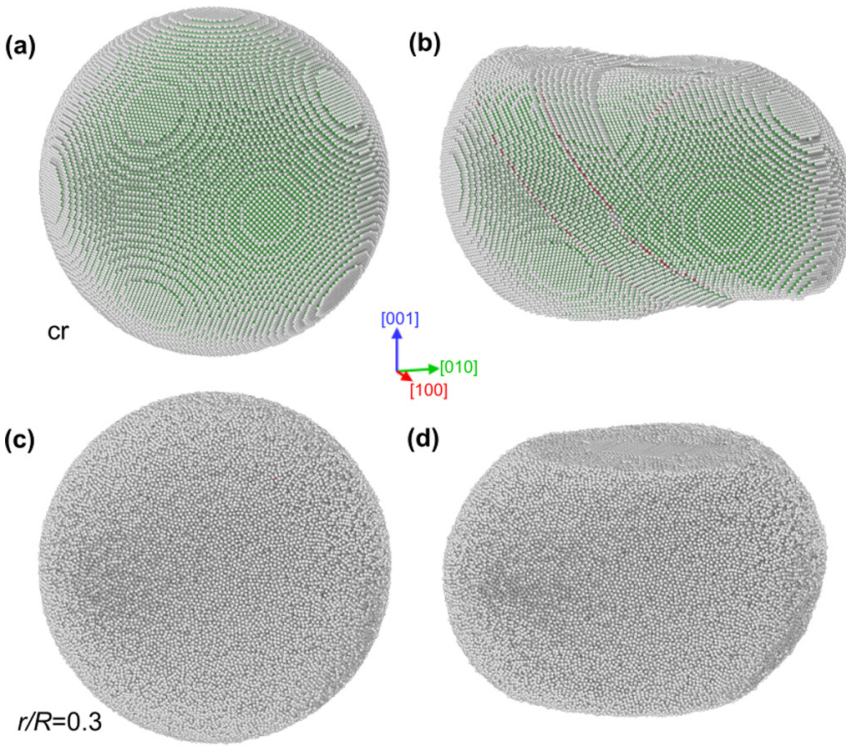

FIG. 10. Evolution of NPs external shape during compression. (a) and (b) refer to the purely crystalline $20 \mathrm{~nm} \mathrm{Ni} \mathrm{NP,} \mathrm{(c)} \mathrm{and}$ (d) refer to the $r / R=0.3 \mathrm{cr}$ - $a$ NP. Snapshots are performed before compression [(a), (c)] and at $\epsilon=0.3[(\mathrm{~b}),(\mathrm{d})]$.

surface layer influence both the contact surface area as well as the global shape of the NPs. The influence of the amorphous surface layer on the resulting shape and exposed surfaces of NPs after deformation is of primary importance for several fields of application such as the elaboration and processing of materials by, e.g., spark plasma sintering and hot pressing approaches [59-61], or the lubrication of mechanical systems $[7,62,63]$, where strong deformation of NPs can occur.

Finally, while all investigated NPs are characterized by comparable contact areas up to $\epsilon=8 \%$, the indenter force of the $c r$-NP [Fig. 9(a)] is significantly larger, which leads to a larger effective Young's modulus compared to the $c r-a$ systems (260 GPa versus $120 \mathrm{GPa}$ ). Although the observed quantitative difference between the elastic modulus is larger than expected as the poor relaxation of the glassy structure might influence the stiffness of the core-shell system, the qualitative decrease of the modulus is in agreement with the mechanical response of crystalline and amorphous metallic systems of the corresponding chemical compositions reported in literature [64-66].

\section{CONCLUSIONS}

This work aimed to investigate the influence of an amorphous surface layer on the mechanical properties and deformation processes of Ni NPs under compression. Accounting for the unstable character of monoatomic metallic glasses, the transferability of the three different EAM potentials (AMB-95 [32], Mish-04 [33], and Mend-12 [34]) to model monoatomic $\mathrm{Ni}$ glass structure and provide a reasonably slow recrystallisation rate of the amorphous surface layer surrounding the FCC NPs core was investigated. Mend-12 provides the amorphous structure with the lowest SRO and the fastest crystallization rate, while Mish-04 provides the slowest crystallization rate during both sample fabrication and compression processes.

During compression, the amorphous surface layer drastically reduces the effective elastic modulus of the NPs, and leads to a delayed onset of dislocation nucleation when compared to its crystalline counterpart. The shape and contact surfaces of the NPs are also strongly influenced by the amorphous surface layer, which reduces the stress response during plastic deformation. While the amorphous surface layer is characterized by homogeneous shear, the crystalline core of the particles exhibits IDN of Shockley partial dislocations, which can further lead to the formation of nanotwins interacting with each other in a complex manner. No pyramidal dislocation structures (characteristic for $\langle 001\rangle$-oriented FCC NPs) were observed in the NPs with an amorphous surface layer. Thus, in this study we show that besides the mechanical response, the presence of an amorphous surface layers affects the elementary deformation processes and the shape of the NPs under compression. This study brings new perspectives on the deformation of nano-objects with amorphized/oxidized surface, and might help to interpret recent experiments such as in situ TEM nanocompression tests.

\section{ACKNOWLEDGMENTS}

This work was financially supported by the young researcher grant "Projet inter-équipe jeune chercheur 2017" from MATEIS, INSA-Lyon. The HPC resources (P2CHPD) were provided by the Fédération Lyonnaise de Modélisation et Sciences Numériques (FLMSN), partner of the EQUIPEX EQUIP@MESO. The authors thank two anonymous reviewers for their insightful comments and remarks.
[1] H. G. Craighead, Nanoelectromechanical systems, Science 290, 1532 (2000).

[2] O. Y. Loh and H. D. Espinosa, Nanoelectromechanical contact switches, Nat. Nanotech. 7, 283 (2012).

[3] Y. Liu, X. Sun, S. Wang, M. Xie, A. Chen, and R. Long, Preparation of nanoparticles embedded microcapsules (nems) and their application in drug release, Mater. Lett. 75, 48 (2012).

[4] X. Lin, T. Bai, Y. Y. Zuo, and N. Gu, Promote potential applications of nanoparticles as respiratory drug carrier: Insights from molecular dynamics simulations, Nanoscale 6, 2759 (2014).
[5] P. Alivisatos, The use of nanocrystals in biological detection, Nat. Biotechnol. 22, 47 (2003).

[6] P. D. Howes, R. Chandrawati, and M. M. Stevens, Bionanotechnology. Colloidal nanoparticles as advanced biological sensors, Science 346, 1247390 (2014).

[7] L. Rapoport, Y. Bilik, Y. Feldman, M. Homyonfer, S. R. Cohen, and R. Tenne, Hollow nanoparticles of $\mathrm{WS}_{2}$ as potential solidstate lubricants, Nature 387, 791 (1997).

[8] A. Hernández Battez, R. González, J. L. Viesca, J. E. Fernández, J. M. Díaz Fernández, A. Machado, R. Chou, and J. Riba, 
$\mathrm{CuO}, \mathrm{ZrO}_{2}$ and $\mathrm{ZnO}$ nanoparticles as antiwear additive in oil lubricants, Wear 265, 422 (2008).

[9] D. Mordehai, S.-W. Lee, B. Backes, D. J. Srolovitz, W. D. Nix, and E. Rabkin, Size effect in compression of single-crystal gold microparticles, Acta Mater. 59, 5202 (2011).

[10] R. Maaß, L. Meza, B. Gan, S. Tin, and J. R. Greer, Ultrahigh strength of dislocation-free $\mathrm{Ni}_{3} \mathrm{Al}$ nanocubes, Small 8, 1869 (2012).

[11] J.-J. Bian and G.-F. Wang, Atomistic deformation mechanisms in copper nanoparticles, J. Comput. Theor. Nanosci. 10, 2299 (2013).

[12] I. Issa, J. Amodeo, J. Réthoré, L. Joly-Pottuz, C. Esnouf, J. Morthomas, M. Perez, J. Chevalier, and K. Masenelli-Varlot, In situ investigation of $\mathrm{MgO}$ nanocube deformation at room temperature, Acta Mater. 86, 295 (2015).

[13] Q. Yu, L. Qi, R. K. Mishra, X. Zeng, and A. M. Minor, Size-dependent mechanical properties of $\mathrm{Mg}$ nanoparticles used for hydrogen storage, Appl. Phys. Lett. 106, 261903 (2015).

[14] J. Amodeo and K. Lizoul, Mechanical properties and dislocation nucleation in nanocrystals with blunt edges, Mater. Des. 135, 223 (2017).

[15] A. Sharma, J. Hickman, N. Gazit, E. Rabkin, and Y. Mishin, Nickel nanoparticles set a new record of strength, Nat. Commun. 9, 4102 (2018).

[16] W. Gerberich, E. B. Tadmor, J. Kysar, J. A. Zimmerman, A. M. Minor, I. Szlufarska, J. Amodeo, B. Devincre, E. Hintsala, and R. Ballarini, Review Article: Case studies in future trends of computational and experimental nanomechanics, J. Vac. Sci. Technol. A 35, 060801 (2017).

[17] W.-Z. Han, L. Huang, S. Ogata, H. Kimizuka, Z.-C. Yang, C. Weinberger, Q.-J. Li, B.-Y. Liu, X.-X. Zhang, J. Li, E. Ma, and Z.-W. Shan, From "smaller is stronger" to "sizeindependent strength plateau": Towards measuring the ideal strength of iron, Adv. Mater. 27, 3385 (2015).

[18] F. G. Sen, A. T. Alpas, A. C. T. van Duin, and Y. Qi, Oxidationassisted ductility of aluminium nanowires, Nat. Commun. 5, 3959 (2014).

[19] G. Aral, Y.-J. Wang, S. Ogata, and A. C. T. van Duin, Effects of oxidation on tensile deformation of iron nanowires: Insights from reactive molecular dynamics simulations, J. Appl. Phys. 120, 135104 (2016)

[20] A. C. T. van Duin, S. Dasgupta, F. Lorant, and W. A. Goddard, ReaxFF: A reactive force field for hydrocarbons, J. Phys. Chem. A 105, 9396 (2001)

[21] T. Liang, T.-R. Shan, Y.-T. Cheng, B. D. Devine, M. Noordhoek, Y. Li, Z. Lu, S. R. Phillpot, and S. B. Sinnott, Classical atomistic simulations of surfaces and heterogeneous interfaces with the charge-optimized many body (COMB) potentials, Mater. Sci. Eng. R 74, 255 (2013).

[22] N. Salles, O. Politano, E. Amzallag, and R. Tétot, Molecular dynamics study of high-pressure alumina polymorphs with a tight-binding variable-charge model, Comput. Mater. Sci. 111, 181 (2016).

[23] S. Takeda and J. Yamasaki, Amorphization in Silicon by Electron Irradiation, Phys. Rev. Lett. 83, 320 (1999).

[24] L. Zhong, J. Wang, H. Sheng, Z. Zhang, and S. X. Mao, Formation of monatomic metallic glasses through ultrafast liquid quenching, Nature 512, 177 (2014).
[25] Y. Jing and Q. Meng, Molecular dynamics simulations of the mechanical properties of crystalline/amorphous silicon core/shell nanowires, Physica B: Phys. Condens. Matter 405, 2413 (2010).

[26] J. Guénolé, J. Godet, and S. Brochard, Plasticity in crystallineamorphous core-shell Si nanowires controlled by native interface defects, Phys. Rev. B 87, 045201 (2013).

[27] A. P. Baikov, V. A. Ivanchenko, V. I. Motorin, S. L. Musher, and A. F. Shestak, The one-component metallic glasses from nickel and molybdenum, Phys. Lett. A 113, 38 (1985).

[28] S. Trady, M. Mazroui, A. Hasnaoui, and K. Saadouni, Molecular dynamics study of atomic-level structure in monatomic metallic glass, J. Non-Cryst. Solids 443, 136 (2016).

[29] S. Trady, A. Hasnaoui, M. Mazroui, and K. Saadouni, Local atomic structures of single-component metallic glasses, Eur. Phys. J. B 89, 223 (2016).

[30] A. I. Fedorchenko, On the glass transition of the one-component metallic melts, J. Cryst. Growth 475, 362 (2017).

[31] W. Gonçalves, J. Amodeo, J. Morthomas, P. Chantrenne, M. Perez, G. Foray, and C. L. Martin, Nanocompression of secondary particles of silica aerogel, Scr. Mater. 157, 157 (2018).

[32] J. E. Angelo, N. R. Moody, and M. I. Baskes, Trapping of hydrogen to lattice defects in nickel, Modell. Simul. Mater. Sci. Eng. 3, 289 (1995)

[33] Y. Mishin, Atomistic modeling of the $\gamma$ and $\gamma^{\prime}$-phases of the Ni-Al system, Acta Mater. 52, 1451 (2004).

[34] M. I. Mendelev, M. J. Kramer, S. G. Hao, K. M. Ho, and C. Z. Wang, Development of interatomic potentials appropriate for simulation of liquid and glass properties of $\mathrm{NiZr}_{2}$ alloy, Philos. Mag. 92, 4454 (2012).

[35] S. Plimpton, Fast parallel algorithms for short-range molecular dynamics, J. Comput. Phys. 117, 1 (1995).

[36] W. G. Hoover, Canonical dynamics: Equilibrium phase-space distributions, Phys. Rev. A 31, 1695 (1985).

[37] P. Hirel, Atomsk: A tool for manipulating and converting atomic data files, Comput. Phys. Commun. 197, 212 (2015).

[38] A. Stukowski, Visualization and analysis of atomistic simulation data with OVITO the open visualization tool, Modell. Simul. Mater. Sci. Eng. 18, 015012 (2010).

[39] G. J. Ackland and A. P. Jones, Applications of local crystal structure measures in experiment and simulation, Phys. Rev. B 73, 054104 (2006)

[40] A. France-Lanord, E. Blandre, T. Albaret, S. Merabia, D. Lacroix, and K. Termentzidis, Atomistic amorphous/ crystalline interface modeling for superlattices and core/ shell nanowires, J. Phys.: Condens. Matter 26, 055011 (2014).

[41] A. France-Lanord, S. Merabia, T. Albaret, D. Lacroix, and K. Termentzidis, Thermal properties of amorphous/crystalline silicon superlattices, J. Phys.: Condens. Matter 26, 355801 (2014)

[42] S. Das and A. Dutta, Elastic behavior of amorphous-crystalline silicon nanocomposite: An atomistic view, Physica E 85, 180 (2017).

[43] H. Edelsbrunner and E. P. Mücke, Three-dimensional alpha shapes, ACM Trans. Graph. 13, 43 (1994).

[44] A. Stukowski, Computational analysis methods in atomistic modeling of crystals, JOM 66, 399 (2014). 
[45] A. Posada-Amarillas and I. L. Garzón, Microstructural analysis of simulated liquid and amorphous Ni, Phys. Rev. B 53, 8363 (1996).

[46] T. Ichikawa, Electron diffraction study of the local atomic arrangement in amorphous iron and nickel films, Phys. Status Solidi A 19, 707 (1973).

[47] S. B. H. Salah, C. Gerard, and L. Pizzagalli, Influence of surface atomic structure on the mechanical response of aluminum nanospheres under compression, Comput. Mater. Sci. 129, 273 (2017).

[48] D. Kilymis, C. Gerard, J. Amodeo, U. V. Waghmare, and L. Pizzagalli, Uniaxial compression of silicon nanoparticles: An atomistic study on the shape and size effects, Acta Mater. 158, 155 (2018).

[49] D. Mordehai, E. Rabkin, and D. J. Srolovitz, Pseudoelastic Deformation During Nanoscale Adhesive Contact Formation, Phys. Rev. Lett. 107, 096101 (2011).

[50] S. Korte, J. S. Barnard, R. J. Stearn, and W. J. Clegg, Deformation of silicon - insights from microcompression testing at 25-500 ${ }^{\circ} \mathrm{C}$, Int. J. Plast. 27, 1853 (2011).

[51] R. Soler, J. Mikel Molina-Aldareguia, J. Segurado, and J. Lorca, Effect of misorientation on the compression of highly anisotropic single-crystal micropillars, Adv. Eng. Mater. 14, 1004 (2012).

[52] C. Goldenberg, A. Tanguy, and J.-L. Barrat, Particle displacements in the elastic deformation of amorphous materials: Local fluctuations vs non-affine field, Europhys. Lett. 80, 16003 (2007).

[53] A. Tanguy, F. Leonforte, and J.-L. Barrat, Plastic response of a 2D Lennard-Jones amorphous solid: Detailed analysis of the local rearrangements at very low strain rate, Eur. Phys. J. E 20, 355 (2006).

[54] C. Fusco, T. Albaret, and A. Tanguy, Role of local order in the small-scale plasticity of model amorphous materials, Phys. Rev. E 82, 066116 (2010).

[55] F. Shimizu, S. Ogata, and J. Li, Yield point of metallic glass, Acta Mater. 54, 4293 (2006).
[56] F. Shimizu, S. Ogata, and J. Li, Theory of shear banding in metallic glasses and molecular dynamics calculations, Mater. Trans. 48, 2923 (2007).

[57] Y. Q. Cheng, A. J. Cao, H. W. Sheng, and E. Ma, Local order influences initiation of plastic flow in metallic glass: Effects of alloy composition and sample cooling history, Acta Mater. 56, 5263 (2008).

[58] Y. Wang, J. Li, A. V. Hamza, and T. W. Barbee, Ductile crystalline-amorphous nanolaminates, Proc. Natl. Acad. Sci. USA 104, 11155 (2007).

[59] Y. Lan, A. J. Minnich, G. Chen, and Z. Ren, Enhancement of thermoelectric figure-of-merit by a bulk nanostructuring approach, Adv. Funct. Mater. 20, 357 (2010).

[60] R. Chaim, Densification mechanisms in spark plasma sintering of nanocrystalline ceramics, Mater. Sci. Eng. A 443, 25 (2007).

[61] O. Guillon, J. Gonzalez-Julian, B. Dargatz, T. Kessel, G. Schierning, J. Räthel, and M. Herrmann, Field-assisted sintering technology/spark plasma sintering: Mechanisms, materials, and technology developments, Adv. Eng. Mater. 16, 830 (2014).

[62] A. Katz, M. Redlich, L. Rapoport, H. D. Wagner, and R. Tenne, Self-lubricating coatings containing fullerene-like $\mathrm{WS}_{2}$ nanoparticles for orthodontic wires and other possible medical applications, Tribol. Lett. 21, 135 (2006).

[63] O. Tevet, P. Von-Huth, R. Popovitz-Biro, R. Rosentsveig, H. D. Wagner, and R. Tenne, Friction mechanism of individual multilayered nanoparticles, Proc. Natl. Acad. Sci. USA 108, 19901 (2011).

[64] M. F. Ashby, A. N. Nelson, and R. M. A. Centamore, The mechanical properties of a glassy metal; $\mathrm{Ni}_{3} \mathrm{P}$, Scr. Metall. 4 , 715 (1970).

[65] J. M. Pelletier, Dynamic mechanical properties in a $\mathrm{Zr}_{46.8} \mathrm{Ti}_{13.8} \mathrm{Cu}_{12.5} \mathrm{Ni}_{10} \mathrm{Be}_{27.5}$ bulk metallic glass, J. Alloys Compd. 393, 223 (2005).

[66] D. J. Safarik and R. B. Schwarz, Elastic constants of amorphous and single-crystal $\mathrm{Pd}_{40} \mathrm{Cu}_{40} \mathrm{P}_{20}$, Acta Mater. 55, 5736 (2007). 